\title{
New Perspectives on Graphene/ Polymer Fibers and Fabrics for Smart Textiles: The Relevance of the Polymer/Graphene Interphase
}

\author{
Horacio J. Salavagione*, Marián A. Gómez-Fatou, Peter S. Shuttleworth and Gary J. Ellis
}

Departamento de Física de Polímeros, Elastómeros y Aplicaciones Energéticas, Instituto de Ciencia y Tecnología de Polímeros (ICTP-CSIC), Madrid, Spain

The fast-growing interest in smart textiles for wearable electronics or sensors is stimulating considerable activity in the development of functional fibers and fabrics that incorporate graphene, due to its outstanding electrical, mechanical, and thermal properties, among others. This paper provides an overview of the current state-of-the-art of research in this field, and a perspective on the factors decisive to its growth, in particular

OPEN ACCESS

Edited by:

Emilia Morallon

University of Alicante, Spain

Reviewed by:

Joaquin Arias-Pardilla,

Universidad Politécnica de

Cartagena, Spain

Juan Manuel Sieben,

Universidad Nacional del Sur,

Argentina

*Correspondence:

Horacio J. Salavagione

horacio@ictp.csic.es

Specialty section:

This article was submitted to

Carbon-Based Materials,

a section of the journal

Frontiers in Materials

Received: 19 December 2017

Accepted: 12 March 2018

Published: 28 March 2018

Citation:

Salavagione HJ, Gómez-Fatou MA, Shuttleworth PS and Ellis GJ (2018)

New Perspectives on Graphene/

Polymer Fibers and Fabrics for Smart

Textiles: The Relevance of the

Polymer/Graphene Interphase.

Front. Mater. 5:18.

doi: 10.3389/fmats.2018.00018 the polymer-graphene interphase.

Keywords: nanocomposites, 2D materials, flexible conductors, wearable devices, processing

\section{INTRODUCTION}

Smart textiles is a broad generic term for materials that have been woven like typical fabrics but can integrate advanced functions such as energy storage and conversion (Weng et al., 2016), sensors (Lee et al., 2016), color change (Gauvreau et al., 2008), drug release (Yetisen et al., 2016), or can deliver responses to external stimuli, among others. Smart textiles are fabricated by the incorporation of functional components into conventional textile regimes (Hansora et al., 2015) offering platforms sensitive to mechanical, electrical, thermal, optical, or magnetic stimuli. Graphene can be considered an interesting candidate as the functional component in smart textiles due to its outstanding properties (electrical, mechanical, thermal, optical, etc.), and its incorporation into synthetic and natural textiles could help pave the way for the preparation of multifunctional textiles for wider implementation. The introduction of graphene-based smart materials in the market will depend fundamentally on the methodologies developed to incorporate graphene into textile devices. In this respect, there has been much research directed toward the fabrication of graphene fibers (GrFs) (Meng et al., 2015; Xu and Gao, 2015), which exhibit very high-electrical conductivities, but have the drawback of poor and unreliable mechanical performance. Thus, combination of graphene with the polymer fibers is an alternative approach to enhance mechanical strength, toughness and flexibility during use, and deformation.

In this perspective, we will focus on smart textiles based on polymer/graphene composite fibers considering the two main approaches employed to form these materials. These are based on the incorporation of graphene before and/or after processing of the polymeric material, and are divided into (i) mixing graphene with polymers followed by fiber formation (pre-processing) and (ii) graphene (polymer) coating/impregnation of processed polymer (graphene) fibers and fabrics (post-processing).

It is our view that this topic has not yet been addressed from a molecular standpoint and there is very little information available on the nanomaterial/polymer interphase. The absence of strong interactions between the fibers and graphene leads to an inevitable deterioration of the target 
function before the projected end of life of the product. This can be due to a difference in the elasticity coefficient between the polymer and graphene, leading to crack formation via twisting, stretching, or bending during use and laundering. In fact, one of the main challenges of the smart textile industry relates to current methodologies used to functionalize the fibers without detriment to the conductivity (Yetisen et al., 2016). Thus, the main motivation for this perspective is to highlight the importance of designing strategies to enhance the interphase strength in graphene/polymer composite fibers in order to retain the imparted functionality during its lifetime. In this respect, the advances in smart textiles composed of natural and synthetic fibers with functional materials will progress alongside developments in the polymer nanocomposite field.

\section{CURRENT RESEARCH STATUS}

Smart textiles based on natural and synthetic fabrics and graphene have been prepared mostly either by mixing graphene with polymers and subsequently forming the fiber or by coating or impregnating already-processed polymer fibers and fabrics with graphene.

\section{Polymer/Graphene Composite Fiber Formation (Pre-Processing Methods)}

An emerging approach for the preparation of smart textiles consists in mixing an insulating polymer with functional components like conducting polymers (Seyedin et al., 2015), metal nanowires (Yoon et al., 2015), or carbon nanostructures (Liu et al., 2015; Di et al., 2016) and their subsequent integration into fabrics. However, the rigid polymer backbone of conducting polymers makes their processing difficult, resulting in poor longterm stability, whilst the addition of metals can present toxicity issues and adds weight to the fabrics. On the other hand, the addition of graphene can impart flexibility, high modulus, and electrical conductivity, without the disadvantage of additional weight. Different strategies have been used to integrate graphene with polymers during the manufacturing of the fibers. The main objective is to simultaneously attain both the mechanical and electrochemical properties required for a particular smart textile application.

The simplest fabrication method comprises the incorporation of graphene into the polymer solution or melt phase and then the preparation of composite fibers by wet spinning, electrospinning, or melt spinning. Polyvinyl alcohol (PVA)/pristine graphene fibers prepared by electrospinning, using an aqueous solution of polyvinylpyrrolidone to stabilize the graphene dispersion, demonstrated extraordinary enhancements in modulus (Das et al., 2013). Improved mechanical performance was also obtained for polycaprolactone (PCL)/graphene oxide (GO) and PCL/reduced GO (rGO) electrospun nanofibers in which orientation played an important role (Ramazani and Karimi, 2015). Conducting nanofibers of polyaniline/polyethylene oxide with non-covalently functionalized graphene, also fabricated by electrospinning exhibited two orders of magnitude enhancement in electrical conductivity (Moayeri and Ajji, 2015). Wet spinning has been applied to elastomers, and both conducting and insulating polymers have been prepared using mainly GO as an additive, followed by post-spinning reduction (Ding et al., 2014; Chen et al., 2016; Seyedin et al., 2016). PVA/rGO and polypyrrole/rGO fibers prepared by this method showed excellent electrochemical performance for use in lightweight supercapacitors (Ding et al., 2014; Chen et al., 2016). Melt spinning was used to fabricate conducting textile fibers of polypropylene (PP) with hybridized graphite nanoplatelets, carbon black filler, and amine functionalized graphene/polyamide 6 fibers (Nilsson et al., 2013; Hou et al., 2014).

A major challenge in this field is to develop wearable energy storage and conversion devices, with fiber-based supercapacitors being ideal candidates for this purpose. Most of the research in this area has been undertaken by infiltrating GrFs with polymers. Meng et al. fabricated flexible core-shell graphene/conducting polymer fibers using a simple and efficient strategy (Meng et al., 2017). The GrFs were initially prepared via a one-step hydrothermal strategy, and subsequently poly(3,4-ethyledioxythiophene) (PEDOT) was incorporated into the GrFs via in situ interfacial polymerization. These fibers showed excellent electrochemical properties and sufficient modular flexibility to be woven into cloth-like structures. In addition, when incorporated into a solidstate fiber supercapacitor, they showed better capacitive behavior and higher current densities compared with those based on GrFs alone. A fiber supercapacitor with higher energy density was fabricated based on hollow $\mathrm{rGO} /[\mathrm{PEDOT}$ :poly(styrenesulfonate) (PSS)] composite fibers (Qu et al., 2016). The hollow structure was prepared using a solution of GO, PEDOT:PSS, and vitamin $\mathrm{C}$ to reduce $\mathrm{GO}$ in which the $\mathrm{rGO}$ sheets formed lyotropic nematic crystals. A recent advancement has been the development of a stretchable and self-healable supercapacitors produced by wrapping rGO-based composite fibers with an outer shell of carboxylated polyurethane (Figures 1A-F) (Wang et al., 2017).

However, all these pre-mixing methods, which include efficient graphene dispersion and alignment into the fibers and, in some cases, chemical, or thermal reduction, result in a complicated manufacturing process that ultimately limits their industrial viability (Yun et al., 2017).

\section{Post-Processing Coatings}

An approach to avoid the problems associated with pre-mixing consists in impregnating or coating the surface of commercial fibers or textiles with graphene or its derivatives from dispersions and inks. This route is essentially simpler and easier to implement, involving low-temperature processes (especially when pristine graphene dispersions are used), is economically more feasible at an industrial scale, and is an adopted technology already employed.

Due to its better solubility than graphene, GO has been used to form coatings on polymeric fibers and fabrics from water and organic solvents, followed by conversion to rGO. This protocol was employed to produce rGO-based textiles by coating nylon, cotton, and polyester fabrics (Yun et al., 2013). The nylon fabrics were initially covered with bovine serum albumin, which acts as an adhesive, improving the adsorption of GO by electrostatic interactions, and $\mathrm{rGO}$ was obtained by chemical reduction with 

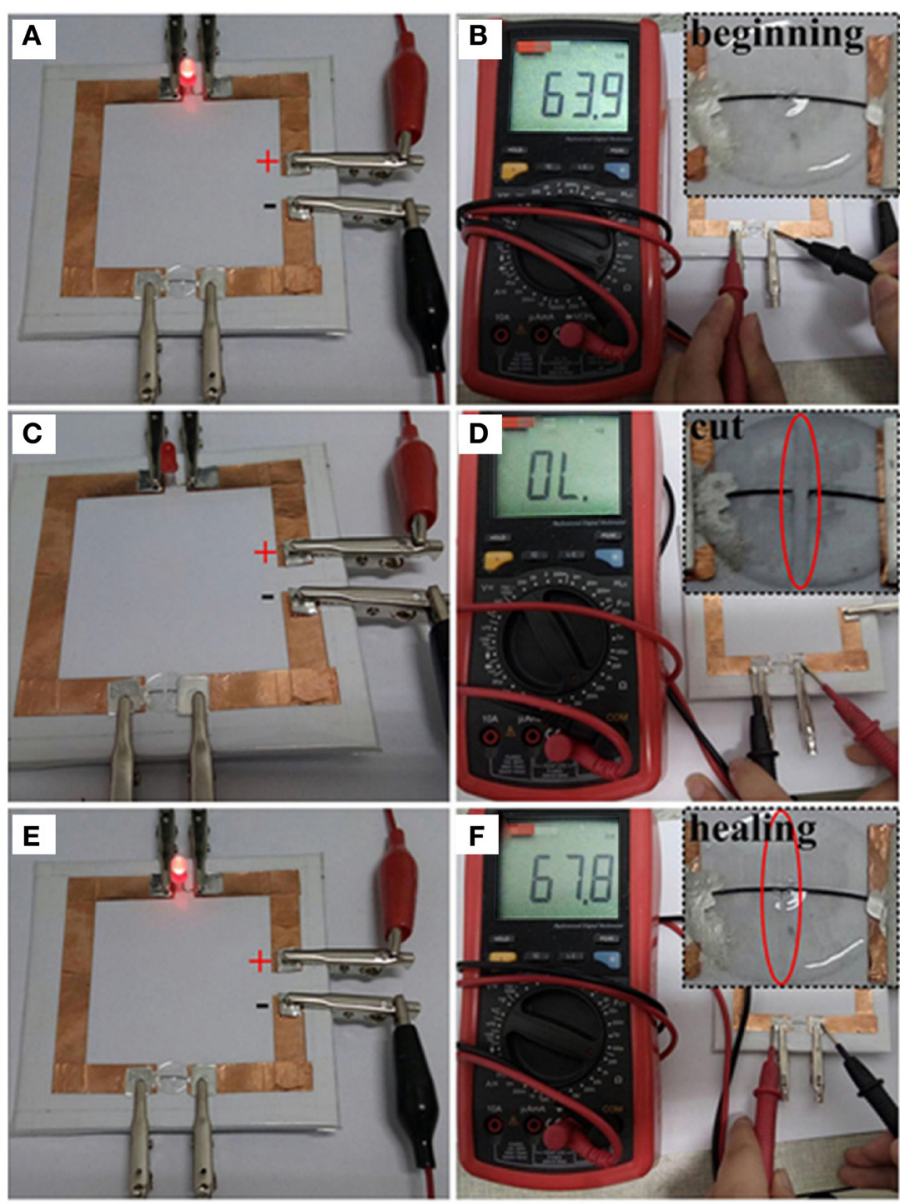

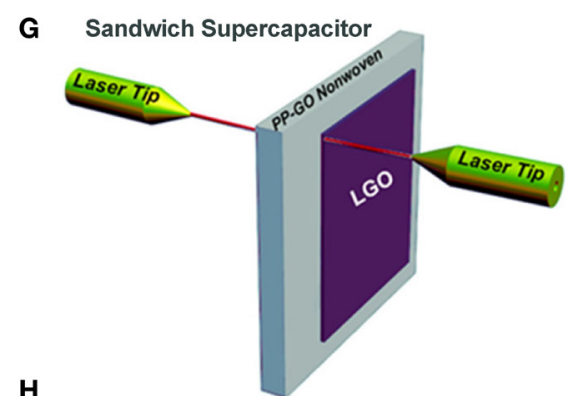

$\mathbf{H}$
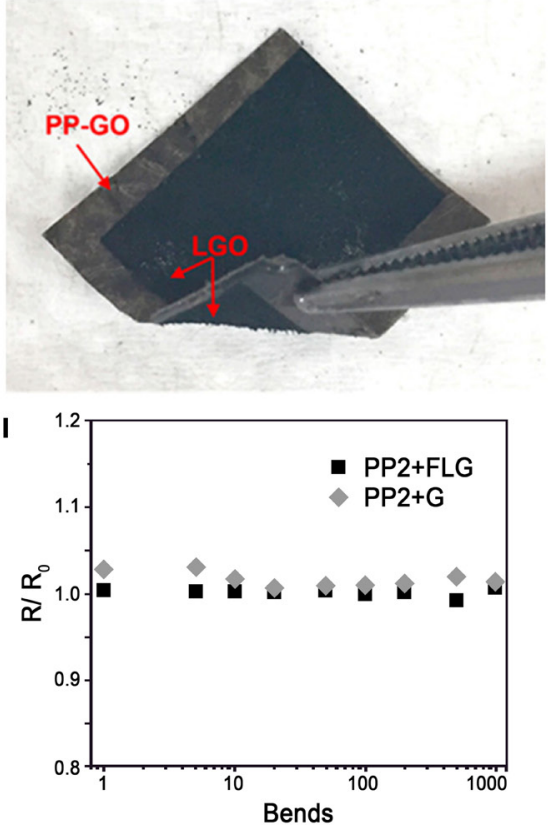

FIGURE 1 | (A-F) Self-healing properties of reduced graphene oxide-based composite fibers with polyurethane. Photographs of the fiber electrode within a circuit with a LED $(\mathbf{A}, \mathbf{C}, \mathbf{E})$ and electrical resistance (B,D,F) of the fiber electrode before breaking, after breaking, and after healing. Adapted with permission from Wang et al. (2017) with permission from The American Chemical Society. (G,H) Scheme of laser-patterning process to fabricate monolithic sandwich supercapacitor on non-woven polypropylene (PP)-GO (G) and photograph of the PP-GO supercapacitor (H). Adapted from Pan et al. (2017) with permission from The American Chemical Society. (I) Variation of resistance during bending cycles with bending radius of $5 \mathrm{~mm}$ for PP fabrics covered with monolayer (PP2 + G) and few-layer graphene (PP2 + FLG). Adapted from Neves et al. (2017) with permission from Nature Publishing Group.

hydroiodic acid (HI). The materials manifested electrical conductivity that is stable after several cycles of mechanical deformation, bending, and washing. A similar approach has been recently reported, where melt blown PP non-woven fabrics are coated with GO from $\mathrm{N}, \mathrm{N}$-dimethylformamide dispersions, followed by reduction with HI (Pan et al., 2017). The non-woven composites formed showed good electrical conductivities and could be used to prepare sandwich supercapacitors via laser-patterning (Figures 1G,H). Similar strategies have also been used to add metal particles to the polymer fibers conducting layer ( $\mathrm{Pu}$ et al., 2016; Babaahmadi et al., 2017).

However, chemical reduction is not appealing from an application point of view and as such, thermal reduction has also been proposed. Torrisi et al. (Ren et al., 2017) infiltrated cotton fabrics with GO dispersions by vacuum filtration and then reduced them by hot pressing at $180^{\circ} \mathrm{C}$. The produced textiles maintained their conductivity after a few washing cycles and also demonstrated potential as strain sensors, retaining this property for more than 400 bending cycles.

Due to the poorer properties of rGO compared with graphene and the disadvantages of additional reduction steps, non-oxidized graphene derivatives, which in principle are harder to process, are proposed. In this context, cotton fabric was covered with graphene nanoribbons (GNR) by dipping it into a water/surfactant GNR dispersion (Gan et al., 2015). The fabric showed improved thermal stability, mechanical properties, and electrical conductivity after 200 bending cycles. Another approach consisted in transferring monolayer and few-layer graphene, obtained by chemical vapor deposition (CVD) onto PP, polyethylene, Nylon, and poly(lactic acid) (Neves et al., 2017). The materials showed very high-surface conductivity, which remained stable after 1,000 cycles of bending (Figure 1I). However, this strategy is not ideal for commercial production of smart textiles due to specific equipment requirements. Also, a CVD-grown graphene mesh has been 
used to cover polymers to form transparent and flexible touch sensors (Kang et al., 2017). Nevertheless, this adds significantly to the cost and is environmentally questionable due to the extra process steps needed to prepare CVD graphene, and the need for harsh chemicals to remove the metal, respectively. In addition, effective transfer of CVD-grown graphene onto the fabric is inherently conditioned by poor adhesion due to the roughness of the substrate.

Beyond the examples described, other strategies based on layer-by-layer self-assembly (Tian et al., 2016; Li et al., 2017), or printing have also been employed (Amr et al., 2017; Gao et al., 2017), the latter to a lesser extent, probably due to crack formation where the structure deforms and to the porous nature of textiles (Jin et al., 2017).

\section{THE ROLE OF CHEMISTRY TO MODULATE THE INTERPHASE}

In graphene-based polymer nanocomposites containing components with very different but complementary properties, the principal factor to obtain materials with superior performance resides in the nature of the polymer/graphene interphase. The stronger the interphase the better the transfer of properties between components. Therefore, much effort has been devoted to control the graphene/polymer interphase. Polymer nanocomposites have evolved from the initial simple mixing of components to the implementation of complex chemical routes and processing strategies that have resulted in near optimal transfer of the filler properties of toughness, electrical conductivity, thermal stability, etc., whilst maintaining the versatility and ease of processing of the chosen polymer. Thus, we are convinced that the field of smart textiles based on polymer fibers and graphene is likely to advance in parallel with the polymer nanocomposites field. In this respect, it is important to point out that the latter is still in an early stage of progress and substantial advances and developments can be envisaged in the coming years. Among these, the most effective improvements will stem from further understanding and precise control of the graphene/fiber interphase.

It is also suggested that research and production of polymeric textiles coated with conductive graphene layers can take advantage of the molecular-level design of bulk polymer nanocomposites, particularly with a view to property retention after mechanical deformation (strain, bending, and even twisting) and washing cycles. To the best of our knowledge, to date only a couple of examples of chemical functionalization of graphene and its derivatives for smart textiles and wearable electronics have been reported

\section{A}

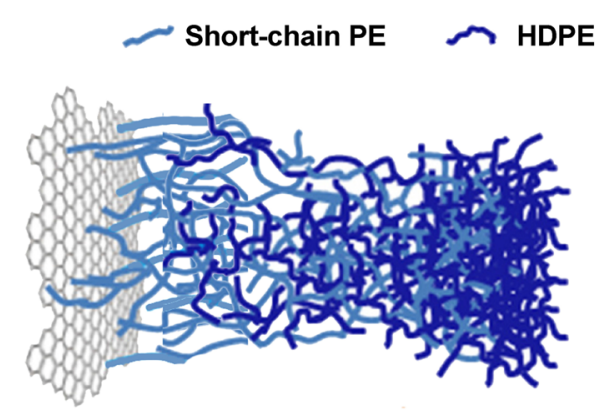

B

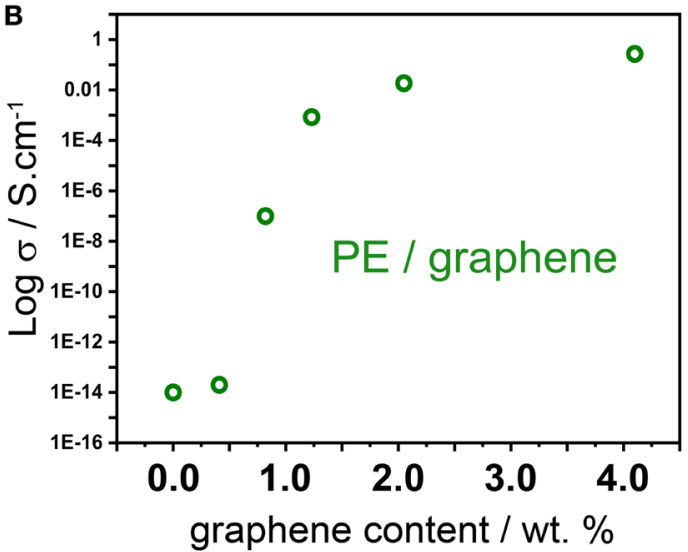

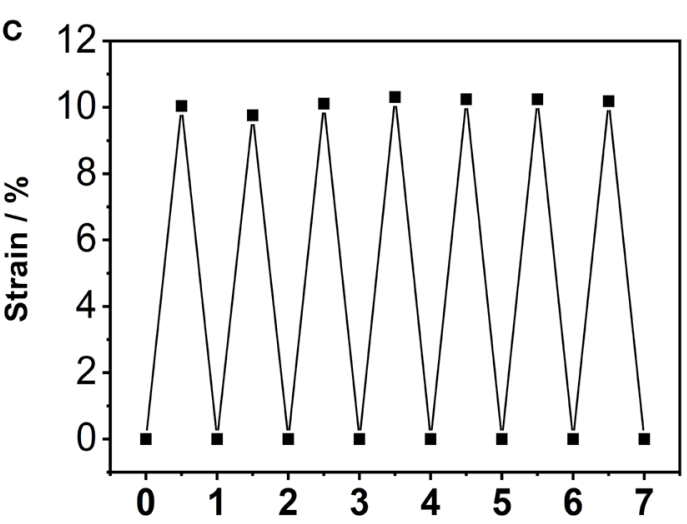

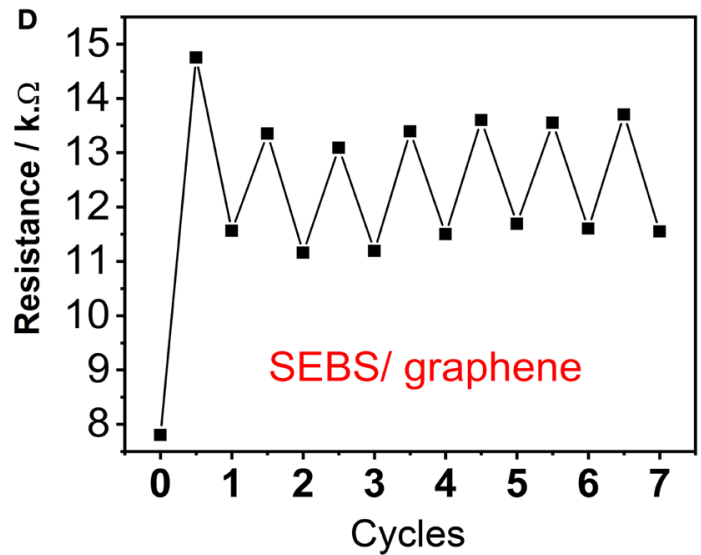

FIGURE 2 | The effect of the functionalization of graphene with polymer brushes on the properties of its nanocomposites. (A) Schematic representation of the polymer/graphene interphase for graphene modified with polymer brushes. (B) Electrical percolation of graphene/polyethylene nanocomposites (Castelaín et al., 2013a), reproduced by permission of The Royal Society of Chemistry. (C,D) Electromechanical behavior of nanocomposites of graphene with poly (styrene-bethylene-co-butylene-b-styrene) (SEBS). Reproduced from Enrique-Jimenez et al. (2017) with permission from Elsevier. 
(Hou et al., 2014; Zarrin et al., 2016). In the last few years, we have focused our investigation on the design of specific synthetic protocols for graphene functionalization, where the chosen chemical modification is designed around the target polymer where graphene is to be incorporated. These approaches have progressed from functionalization with discrete molecules (Salavagione et al., 2009b; Coşkun et al., 2012; Castelaín et al., 2013c), to the covalent bonding of graphene to polymers (Salavagione et al., 2009a; Salavagione and Martínez, 2011; Castelaín et al., 2012) and the functionalization of graphene with short polymer brushes (Castelaín etal.,2013a,b; Salavagione, 2014; Quiles-Diaz etal., 2016; Salavagione et al., 2016; Enrique-Jimenez et al., 2017; Quiles-Díaz et al., 2017). The former method uses simple chemical reactions and can lead to good functionalization levels. Among the most important reactions used, it its worth mentioning the oxidation of graphene to furnish it with polar moieties (hydroxyl, epoxide, carboxylic, etc.) and coupling reactions with diazonium salts that has allowed the modification of graphene with aniline derivatives such as sulfanilic acid, amino-4-hydroxy-2-naphthalenesulfonic, aminobenzoic acid, p-aminophenol, p-aminothiophenol, and 4-ethynylaniline, to name a few. However, this strategy generates discrete supramolecular graphene/fiber interactions that could be unstable to washing or deformation cycles. The covalent graphene/ polymer connection generates the strongest interphase, but the degree of modification is too low due to steric factors, which may lead to incomplete coverage of the textile fibers. Using this approach, mainly polar polymers, such as poly(vinyl alcohol), poly(acrylic acid), poly(acryl amide), and poly( $N$-isopropylacrylamide), have been covalently bonded to graphene. The functionalization of graphene with low-molecular weight polymers represents an intermediate strategy that can circumvent the drawbacks of the former approaches (Figure 2A). Firstly, the degree of graphene functionalization is much higher than in the case of the covalent approach. And secondly, although the graphene/polymer fiber

\section{REFERENCES}

Amr, M. A., Nazmul, K., Cristina, V., Shaila, A., Kostya, S. N., and Stephen, G. Y. (2017). Ultraflexible and robust graphene supercapacitors printed on textiles for wearable electronics applications. 2D Materials 4, 035016. doi:10.1088/20531583/aa7d71

Babaahmadi, V., Montazer, M., and Gao, W. (2017). Low temperature welding of graphene on PET with silver nanoparticles producing higher durable electroconductive fabric. Carbon N. Y. 118, 443-451. doi:10.1016/j.carbon.2017. 03.066

Castelaín, M., Martínez, G., Ellis, G., and Salavagione, H. J. (2013a). Versatile chemical tool for the preparation of conductive graphene-based polymer nanocomposites. Chem. Commun. 49, 8967-8968. doi:10.1039/C3CC43729A

Castelaín, M., Martínez, G., Marco, C., Ellis, G., and Salavagione, H. J. (2013b). Effect of Click-chemistry approaches for graphene modification on the electrical, thermal, and mechanical properties of polyethylene/graphene nanocomposites. Macromolecules 46, 8980-8987. doi:10.1021/ma401606d

Castelaín, M., Shuttleworth, P. S., Marco, C., Ellis, G., and Salavagione, H. J. (2013c). Comparative study of the covalent diazotization of graphene and carbon nanotubes using thermogravimetric and spectroscopic techniques. Phys. Chem. Chem. Phys. 15, 16806-16811. doi:10.1039/c3cp52672k

Castelaín, M., Martínez, G., Merino, P., Martín-Gago, J. Á, Segura, J. L., Ellis, G., et al. (2012). Graphene functionalisation with a conjugated poly(fluorene) by click coupling: striking electronic properties in solution. Chemistry 18, 4965-4973. doi:10.1002/chem.201102008 interphase is governed by supramolecular interactions, the density of these interactions is very high, minimizing problems generated by deformation and washing. This approach has allowed us to obtain graphene-based nanocomposites, with the most widely employed thermoplastic polymers [high-density polyethylene, isotactic PP, polystyrene, and poly(styrene-b-ethylene-co-butylene-b-styrene)], which display superior mechanical, electrical, and electromechanical properties (Figures 2B-D). Nonetheless, it is likely that all of the above strategies will be useful, and the determining factor will depend on the nature of the textile and its final application.

\section{CONCLUDING REMARKS}

This perspective raises the importance of molecular-level control of the polymer graphene interphase with the objective to facilitate the design of efficient approaches for smart textiles fabrication. It is clear that the knowledge generated so far in the field of bulk graphene-based polymer nanocomposites offers a very valuable tool for this purpose and will continue to facilitate advances valuable to the development of graphene-based smart textiles.

\section{AUTHOR CONTRIBUTIONS}

All authors contributed equally to this manuscript.

\section{FUNDING}

Financial support from Spain (grants MAT2013-47898-C2-2-R, MAT2017-88382-P, MAT2014-54231-C4-4-P and MAT201459674-JIN) and CSIC (grant: 201760I084) is gratefully acknowledged. PS gratefully acknowledges the Spanish Ministry of Economy and Competitivity (MINECO) for a Ramon y Cajal Senior Research Fellowship.

Chen, S., Ma, W., Xiang, H., Cheng, Y., Yang, S., Weng, W., et al. (2016). Conductive, tough, hydrophilic poly(vinyl alcohol)/graphene hybrid fibers for wearable supercapacitors. J. Power Sources 319, 271-280. doi:10.1016/j.jpowsour.2016. 04.030

Coşkun, E., Zaragoza-Contreras, E. A., and Salavagione, H. J. (2012). Synthesis of sulfonated graphene/polyaniline composites with improved electroactivity. Carbon N. Y. 50, 2235-2243. doi:10.1016/j.carbon.2012.01.041

Das, S., Wajid, A. S., Bhattacharia, S. K., Wilting, M. D., Rivero, I. V., and Green, M. J. (2013). Electrospinning of polymer nanofibers loaded with noncovalently functionalized graphene. J. Appl. Polym. Sci. 128, 4040-4046. doi:10.1002/app. 38694

Di, J., Zhang, X., Yong, Z., Zhang, Y., Li, D., Li, R., et al. (2016). Carbon-nanotube fibers for wearable devices and smart textiles. Adv. Mater. Weinheim 28, 10529-10538. doi:10.1002/adma.201601186

Ding, X., Zhao, Y., Hu, C., Hu, Y., Dong, Z., Chen, N., et al. (2014). Spinning fabrication of graphene/polypyrrole composite fibers for all-solid-state, flexible fibriform supercapacitors. J. Mater. Chem. A 2, 12355-12360. doi:10.1039/ c4ta01230e

Enrique-Jimenez, P., Quiles-Díaz, S., Salavagione, H. J., Wesner, D., Schönherr, H., González-Casablanca, J., et al. (2017). Control of the structure and properties of SEBS nanocomposites via chemical modification of graphene with polymer brushes. Eur. Polym. J. 97, 1-13. doi:10.1016/j.eurpolymj.2017.09.047

Gan, L., Shang, S., Yuen, C. W. M., and Jiang, S.-X. (2015). Graphene nanoribbon coated flexible and conductive cotton fabric. Compos. Sci. Technol. 117, 208-214. doi:10.1016/j.compscitech.2015.06.019 
Gao, M., Li, L., and Song, Y. (2017). Inkjet printing wearable electronic devices. J. Mater. Chem. C 5, 2971-2993. doi:10.1039/c7tc00038c

Gauvreau, B., Guo, N., Schicker, K., Stoeffler, K., Boismenu, F., Ajji, A., et al. (2008). Color-changing and color-tunable photonic bandgap fiber textiles. Opt. Express 16, 15677-15693. doi:10.1364/oe.16.015677

Hansora, D. P., Shimpi, N. G., and Mishra, S. (2015). Performance of hybrid nanostructured conductive cotton materials as wearable devices: an overview of materials, fabrication, properties and applications. RSC Adv. 5, 107716-107770. doi:10.1039/c5ra16478h

Hou, W., Tang, B., Lu, L., Sun, J., Wang, J., Qin, C., et al. (2014). Preparation and physico-mechanical properties of amine-functionalized graphene/polyamide 6 nanocomposite fiber as a high performance material. RSC Adv. 4, 4848-4855. doi:10.1039/c3ra46525j

Jin, H., Matsuhisa, N., Lee, S., Abbas, M., Yokota, T., and Someya, T. (2017). Enhancing the performance of stretchable conductors for E-textiles by controlled ink permeation. Adv. Mater. Weinheim 29, 1605848. doi:10.1002/ adma.201605848

Kang, M., Kim, J., Jang, B., Chae, Y., Kim, J.-H., and Ahn, J.-H. (2017). Graphenebased three-dimensional capacitive touch sensor for wearable electronics. ACS Nano 11, 7950-7957. doi:10.1021/acsnano.7b02474

Lee, T., Lee, W., Kim, S.-W., Kim, J. J., and Kim, B.-S. (2016). Flexible textile strain wireless sensor functionalized with hybrid carbon nanomaterials supported $\mathrm{ZnO}$ nanowires with controlled aspect ratio. Adv. Funct. Mater. 26, 6206-6214. doi:10.1002/adfm.201601237

Li, X., Hua, T., and Xu, B. (2017). Electromechanical properties of a yarn strain sensor with graphene-sheath/polyurethane-core. Carbon N. Y. 118, 686-698. doi:10.1016/j.carbon.2017.04.002

Liu, M., Du, Y., Miao, Y.-E., Ding, Q., He, S., Tjiu, W. W., et al. (2015). Anisotropic conductive films based on highly aligned polyimide fibers containing hybrid materials of graphene nanoribbons and carbon nanotubes. Nanoscale 7, 1037-1046. doi:10.1039/c4nr06117a

Meng, F., Lu, W., Li, Q., Byun, J.-H., Oh, Y., and Chou, T.-W. (2015). Graphenebased fibers: a review. Adv. Mater. Weinheim 27, 5113-5131. doi:10.1002/adma. 201501126

Meng, Y., Jin, L., Cai, B., and Wang, Z. (2017). Facile fabrication of flexible coreshell graphene/conducting polymer microfibers for fibriform supercapacitors. RSC Adv. 7, 38187-38192. doi:10.1039/c7ra06641d

Moayeri, A., and Ajji, A. (2015). Fabrication of polyaniline/poly(ethylene oxide)/ non-covalently functionalized graphene nanofibers via electrospinning. Synth. Met. 200, 7-15. doi:10.1016/j.synthmet.2014.12.020

Neves, A. I. S., Rodrigues, D. P., De Sanctis, A., Alonso, E. T., Pereira, M. S., Amaral, V. S., et al. (2017). Towards conductive textiles: coating polymeric fibres with graphene. Sci. Rep. 7, 4250. doi:10.1038/s41598-017-04453-7

Nilsson, E., Oxfall, H., Wandelt, W., Rychwalski, R., and Hagström, B. (2013). Melt spinning of conductive textile fibers with hybridized graphite nanoplatelets and carbon black filler. J. Appl. Polym. Sci. 130, 2579-2587. doi:10.1002/app.39480

Pan, Q., Shim, E., Pourdeyhimi, B., and Gao, W. (2017). Highly conductive polypropylene-graphene nonwoven composite via interface engineering. Langmuir 33, 7452-7458. doi:10.1021/acs.langmuir.7b01508

$\mathrm{Pu}, \mathrm{X}$., Li, L., Liu, M., Jiang, C., Du, C., Zhao, Z., et al. (2016). Wearable self-charging power textile based on flexible yarn supercapacitors and fabric nanogenerators. Adv. Mater. Weinheim 28, 98-105. doi:10.1002/adma.201504403

Qu, G., Cheng, J., Li, X., Yuan, D., Chen, P., Chen, X., et al. (2016). A fiber supercapacitor with high energy density based on hollow graphene/conducting polymer fiber electrode. Adv. Mater. Weinheim 28, 3646-3652. doi:10.1002/ adma.201600689

Quiles-Díaz, S., Enrique-Jimenez, P., Papageorgiou, D. G., Ania, F., Flores, A., Kinloch, I. A., et al. (2017). Influence of the chemical functionalization of graphene on the properties of polypropylene-based nanocomposites. Compos. Part A. Appl. Sci. Manuf. 100, 31-39. doi:10.1016/j.compositesa.2017.04.019

Quiles-Diaz, S., Martinez, G., Gomez-Fatou, M. A., Ellis, G. J., and Salavagione, H. J. (2016). Anhydride-based chemistry on graphene for advanced polymeric materials. RSC Adv. 6, 36656-36660. doi:10.1039/c6ra05498f

Ramazani, S., and Karimi, M. (2015). Aligned poly( $\varepsilon$-caprolactone)/graphene oxide and reduced graphene oxide nanocomposite nanofibers: morphological, mechanical and structural properties. Mater. Sci. Eng. C 56, 325-334. doi:10.1016/j.msec.2015.06.045

Ren, J., Wang, C., Zhang, X., Carey, T., Chen, K., Yin, Y., et al. (2017). Environmentally-friendly conductive cotton fabric as flexible strain sensor based on hot press reduced graphene oxide. Carbon N. Y. 111, 622-630. doi:10.1016/j. carbon.2016.10.045

Salavagione, H. J. (2014). Promising alternative routes for graphene production and functionalization. J. Mater. Chem. A 2, 1738. doi:10.1039/c3ta15455f

Salavagione, H. J., Gómez, M. A., and Martínez, G. (2009a). Polymeric modification of graphene through esterification of graphite oxide and poly(vinyl alcohol). Macromolecules 42, 6331-6334. doi:10.1021/ma900845w

Salavagione, H. J., Martinez, G., and Gomez, M. A. (2009b). Synthesis of poly(vinyl alcohol)/reduced graphite oxide nanocomposites with improved thermal and electrical properties. J. Mater. Chem. 19, 5027-5032. doi:10.1039/b904232f

Salavagione, H. J., and Martínez, G. (2011). Importance of covalent linkages in the preparation of effective reduced graphene oxide-poly(vinyl chloride) nanocomposites. Macromolecules 44, 2685-2692. doi:10.1021/ma102932c

Salavagione, H. J., Quiles-Díaz, S., Enrique-Jimenez, P., Martínez, G., Ania, F., Flores, A., et al. (2016). Development of advanced elastomeric conductive nanocomposites by selective chemical affinity of modified graphene. Macromolecules 49, 4948-4956. doi:10.1021/acs.macromol.6b00490

Seyedin, S., Razal, J. M., Innis, P. C., Jalili, R., and Wallace, G. G. (2016). Compositional effects of large graphene oxide sheets on the spinnability and properties of polyurethane composite fibers. Adv. Mater. Interfaces 3, 1500672. doi:10.1002/admi.201500672

Seyedin, S., Razal, J. M., Innis, P. C., Jeiranikhameneh, A., Beirne, S., and Wallace, G. G. (2015). Knitted strain sensor textiles of highly conductive all-polymeric fibers. ACS Appl. Mater. Interfaces 7, 21150-21158. doi:10.1021/acsami.5b04892

Tian, M., Hu, X., Qu, L., Du, M., Zhu, S., Sun, Y., et al. (2016). Ultraviolet protection cotton fabric achieved via layer-by-layer self-assembly of graphene oxide and chitosan. Appl. Surf. Sci. 377, 141-148. doi:10.1016/j.apsusc.2016.03.183

Wang, S., Liu, N., Su, J., Li, L., Long, F., Zou, Z., et al. (2017). Highly stretchable and self-healable supercapacitor with reduced graphene oxide based fiber springs. ACS Nano 11, 2066-2074. doi:10.1021/acsnano.6b08262

Weng, W., Chen, P., He, S., Sun, X., and Peng, H. (2016). Smart electronic textiles. Angew. Chem. Int. Ed. 55, 6140-6169. doi:10.1002/anie.201507333

Xu, Z., and Gao, C. (2015). Graphene fiber: a new trend in carbon fibers. Mater. Today 18, 480-492. doi:10.1016/j.mattod.2015.06.009

Yetisen, A. K., Qu, H., Manbachi, A., Butt, H., Dokmeci, M. R., Hinestroza, J. P., et al. (2016). Nanotechnology in textiles. ACS Nano 10, 3042-3068. doi:10.1021/ acsnano. 5 b08176

Yoon, S. S., Lee, K. E., Cha, H.-J., Seong, D. G., Um, M.-K., Byun, J.-H., et al. (2015). Highly conductive graphene/Ag hybrid fibers for flexible fiber-type transistors. Sci. Rep. 5, 16366. doi:10.1038/srep16366

Yun, Y. J., Ah, C. S., Hong, W. G., Kim, H. J., Shin, J.-H., and Jun, Y. (2017). Highly conductive and environmentally stable gold/graphene yarns for flexible and wearable electronics. Nanoscale 9, 11439-11445. doi:10.1039/c7nr04384h

Yun, Y. J., Hong, W. G., Kim, W.-J., Jun, Y., and Kim, B. H. (2013). A novel method for applying reduced graphene oxide directly to electronic textiles from yarns to fabrics. Adv. Mater. Weinheim 25, 5701-5705. doi:10.1002/adma.201303225

Zarrin, H., Sy, S., Fu, J., Jiang, G., Kang, K., Jun, Y.-S., et al. (2016). Molecular functionalization of graphene oxide for next-generation wearable electronics. ACS Appl. Mater. Interfaces 8, 25428-25437. doi:10.1021/acsami.6b06769

Conflict of Interest Statement: The authors declare that the research was conducted in the absence of any commercial or financial relationships that could be construed as a potential conflict of interest.

Copyright (๑) 2018 Salavagione, Gómez-Fatou, Shuttleworth and Ellis. This is an open-access article distributed under the terms of the Creative Commons Attribution License (CC BY). The use, distribution or reproduction in other forums is permitted, provided the original author(s) and the copyright owner are credited and that the original publication in this journal is cited, in accordance with accepted academic practice. No use, distribution or reproduction is permitted which does not comply with these terms. 